\title{
Influences of molecular orientations on stimulated emission characteristics of oligofluorene films
}

\author{
Hao-Wu Lin ${ }^{\text {a }}$, Chun-Liang Lin ${ }^{\text {a }}$, Chung-Chih $\mathrm{Wu}^{\mathrm{a}, *}$, \\ Teng-Chih Chao ${ }^{\mathrm{b}}$, Ken-Tsung Wong ${ }^{\mathrm{b}}$ \\ ${ }^{a}$ Department of Electrical Engineering, Graduate Institute of Electro-optical Engineering, and Graduate Institute of Electronics \\ Engineering, National Taiwan University, Taipei 10617, Taiwan, ROC \\ ${ }^{\mathrm{b}}$ Department of Chemistry, National Taiwan University, Taipei 10617, Taiwan, ROC
}

Available online 22 June 2006

\begin{abstract}
Efficient luminescence and interesting thermal properties of oligofluorenes provide the opportunity to investigate influences of molecular orientational distributions on characteristics of stimulated emission in organic semiconducting films. While vacuum-deposited terfluorene films exhibit preferential in-plane molecular orientation, they are reoriented and become isotropic with annealing around or above its glass transition temperature. Studies of stimulated emission (amplified spontaneous emission) show that the anisotropic terfluorene films exhibit higher gain and lower threshold than the isotropic films, due to larger confinement of the waveguided mode and larger cross section of stimulated emission. A net gain as high as $78 \mathrm{~cm}^{-1}$ (at a pump intensity of $\sim 30 \mathrm{~kW} / \mathrm{cm}^{2}$ ) and a threshold as low as $0.7 \mathrm{~kW} / \mathrm{cm}^{2}$ were observed. The results suggest that the gain and threshold of stimulated emission in organic semiconducting films could be substantially improved beyond the isotropic case if molecules and their transition dipole moments can be well aligned with the polarization of stimulated emission yet without causing significant loss and emission quenching.
\end{abstract}

(C) 2006 Elsevier B.V. All rights reserved.

PACS: 81.10.-h; 78.66.Qn

Keywords: Stimulated emission; Amplified spontaneous emission; Gain; Molecular orientation; Anisotropy; Spectroscopic ellipsometry

\section{Introduction}

Organic semiconductors have now found wide applications in electronics and optoelectronics, such as organic light-emitting devices, organic thin-film transistors, and organic solar cells, etc. [1-6]. Since

\footnotetext{
* Corresponding author. Tel.: +88622363 5251x346; fax: +886 223671909.

E-mail address: chungwu@cc.ee.ntu.edu.tw (C.-C. Wu).
}

observations of stimulated emission in luminescent and semiconducting organic thin films [7-13], they have also been extensively explored as a new class of gain media for solid-state lasers or semiconducting lasing devices. Such organic gain media in general have the advantages of high photoluminescence efficiency, ease of processing, versatility in substrates, and compatibility in integration with other optoelectronic devices. Also, with the variety and the generally wide gain spectra of luminescent 
organic semiconductors, they have exhibited stimulated emission at wavelengths spanning the whole visible spectra. These promising characteristics of optically pumped organic lasers have thus led to efforts aiming at achieving electrically pumped organic lasers [14-18]. With continuous efforts in pursuing electrically pumped organic lasing, luminescent organic semiconductors with improved characteristics, such as lower thresholds of stimulated emission, enhanced carrier mobilities, and further improved thermal stability, etc., are constantly desired.

As such, recent findings of some intriguing properties of oligofluorenes suggest that they may be of interest for for studies of stimulated emission [19-25]. Appropriate substitutions on C9 of oligofluorenes could render them very high glass transition temperatures $\left(T_{\mathrm{g}}\right)$ of $>200^{\circ} \mathrm{C}$ and high thin-film photoluminescence (PL) quantum yields of $70-90 \%$ in neat films. They are electrochemically stable as indicated by both reversible oxidation and reduction. Furthermore, they exhibit unusual nondispersive ambipolar carrier-transport properties with carrier mobilities over $10^{-2} \mathrm{~cm}^{2} /(\mathrm{V} \mathrm{s})$ for both holes and electrons. In this work, we have performed investigations on the stimulated emission characteristics of a terfluorene. Furthermore utilizing interesting thermal properties of the terfluorene, influences of thin-film morphologies (molecular orientational distributions) on such characteristics are also studied.

\section{Experimental}

The terfluorene (T3, Fig. 1) investigated in this work was synthesized through the Suzuki coupling reaction of 2,7-diboronic ester of 9,9'-diarylfluorene and 2-bromo-9,9-diarylfluorene in the presence of $\mathrm{Pd}\left(\mathrm{PPh}_{3}\right)_{4}$ and $\mathrm{P}^{t} \mathrm{Bu}_{3}$ catalysts with high yields (75$83 \%$ ). Further details of synthesis and chemical analysis had been previously reported [19]. Synthesized T3 molecules were subject to purification by temperature-gradient sublimation before use in this study. Thermal properties of organic compounds were

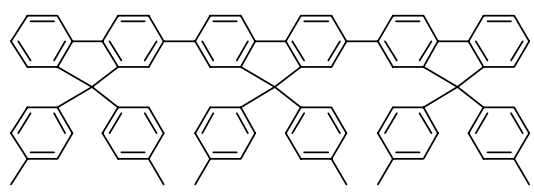

Fig. 1. Molecular structure of T3. investigated by differential scanning calorimetry (DSC).

Thin-film samples of organic compounds for optical characterization were deposited on fused silica substrates by vacuum deposition in a high-vacuum chamber. The deposition was performed at a rate of $2-3 \AA / s$ with the substrate held at room temperature. Thicknesses were monitored using a crystal oscillator during deposition and were verified later with ellipsometry. In some cases, the morphology of thin films was varied by annealing. Annealing of thin-film samples was carried out in a nitrogen atmosphere with a computer-controlled heating stage. After annealing, samples were cooled by contact with a metal block held at the room temperature. The PL quantum yield in thin films was determined using a calibrated integrating sphere system [26]. The $325 \mathrm{~nm}$ line of the $\mathrm{He}-\mathrm{Cd}$ laser was used to excite the thin-film sample placed in the sphere that was coupled to a cooled chargecoupled-device (CCD) spectragraph and was purged with dry nitrogen. The quantum yield was determined by comparing the spectral intensities of the laser source and the sample emission.

Variable-angle spectroscopic ellipsometry (VASE) in the reflection and transmission mode was used to study the optical constants, optical anisotropy, and molecular orientation of oligofluorene films [27-30]. Ellipsometry measures the change in polarization of light as a function of incident angle and wavelength. The experimentally determined ellipsometric values are $\Psi$ and $\Delta$, which are related to the ratio of complex reflection coefficients $\widetilde{R}_{\mathrm{p}}$ and $\widetilde{R}_{\mathrm{s}}$ for p- and s-polarized light, respectively, by $\frac{\widetilde{R}_{\mathrm{p}}}{R_{\mathrm{s}}}=\tan (\Psi) \mathrm{e}^{\mathrm{i} \Delta}$ or the ratio of complex transmission coefficients $\widetilde{T}_{\mathrm{p}}$ and $\widetilde{T}_{\mathrm{s}}$ for $\mathrm{p}$ - and spolarized light, respectively, by $\frac{\widetilde{T}_{\mathrm{p}}}{\widetilde{T}_{\mathrm{s}}}=\tan (\Psi) \mathrm{e}^{\mathrm{i} \Lambda}$. Optical constants of the materials are then determined by first constructing an optical model of the sample with physically meaningful structural and optical parameters, and then by iteratively adjusting these parameters to obtain the best fit to the measured ellipsometric data, i.e., the fitting with the minimum mean square error (MSE).

Ellipsometry over a wavelength range of $270 \mathrm{~nm}-$ $1000 \mathrm{~nm}$ in steps of $5 \mathrm{~nm}$ were performed in air using the J. A. Woollam VASE spectroscopic ellipsometer equipped with a xenon lamp source. The angles of incidence used were between $55^{\circ}$ and $75^{\circ}$ relative to the surface normal in steps of $5^{\circ}$ for reflection ellipsometry and between $40^{\circ}$ and $60^{\circ}$ 
relative to the surface normal in steps of $5^{\circ}$ for transmission ellipsometry. Backside of the fused silica substrate was roughened for reflection ellipsometry to eliminate the incoherent backside reflection from the bottom of the substrate. The nonlinear regression analysis of the measured ellipsometric data was performed using the J. A. Woollam WVASE32 software.

Stimulated emission of T3, in the form of amplified spontaneous emission (ASE), was characterized with films of $\sim 100 \mathrm{~nm}$ thick deposited on the fused silica, which forms a slab waveguide with $\mathrm{SiO}_{2}$ on one side and air on the other. The schematic measurement setup is shown in Fig. 2. The third harmonic of a pulsed $\mathrm{Nd}-\mathrm{YAG}$ laser $(355 \mathrm{~nm}, 10 \mathrm{~ns})$ was used as excitation source for ASE measurements. The energy of pulses was controlled using calibrated neutral density filters. Cylindrical lenses and an adjustable slit were used to shape the excitation beam into a stripe with a width of $1.2 \mathrm{~mm}$ and a length of $4 \mathrm{~mm}$ on the film, creating a stripe-shaped gain region. The uniformity of the intensity and the width of the stripe were confirmed by a beam profiler. In ASE, the spontaneously emitted photons waveguided along the stripe are amplified via stimulated emission as the pumping intensity is increased beyond a certain threshold. As ASE occurs, most of the light is emitted from the end of the stripe and ASE manifests itself with a sudden narrowing of the emission spectrum and an increase in the intensity of edge emission. The edge emission in the plane of the film was detected at one end of the pump stripe using a fiber bundle connected to a cooled charge-coupled-device (CCD) spectrograph.

The variable-stripe-length (VSL) technique was used to measure the net gain of ASE along the waveguide [31-33], as schematically illustrated in Fig. 3. By varying the length of the pump stripe $L$

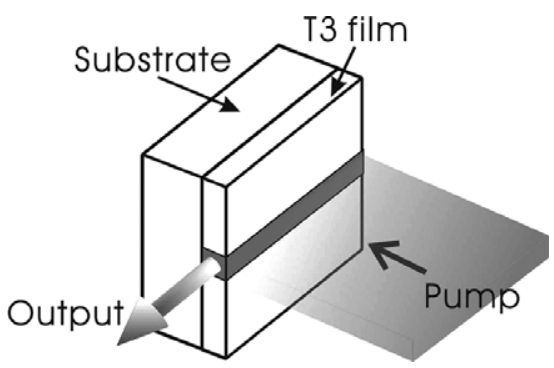

Fig. 2. Schematic setup of ASE measurement.

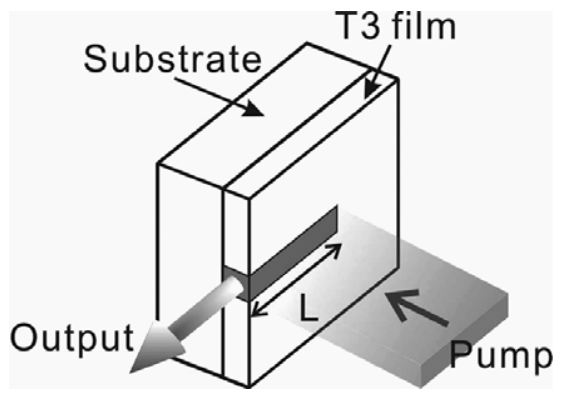

Fig. 3. Schematic setup for measurement of ASE gain.

yet fixing the pumping intensity, one measures the output intensity $I(\lambda)$ of ASE from one end of the stripe as a function of $L$. The output intensity $I(\lambda)$ vs. $L$ follows the dependence of:

$I(\lambda)=\frac{A P_{0}}{g(\lambda)}\left(\mathrm{e}^{g(\lambda) L}-1\right)$

where $A$ is a constant related to the cross section for spontaneous emission, $P_{0}$ is the pump intensity, $g(\lambda)$ is the net gain coefficient, and $L$ is the length of the pump stripe [31-33]. By fitting the experimental data with Eq. (1), one is able to extract the net gain coefficient at the ASE wavelength at a pump intensity.

To measure loss characteristics of the waveguides, the length of the pump stripe was kept constant $(L=2 \mathrm{~mm})$ but the distance of the pump stripe itself from the edge of the sample was varied [31-33], as illustrated in Fig. 4. Assuming the emission intensity from the end of the excitation stripe is $I_{0}$, the emission from the edge of the sample should decrease as $I=I_{0} \exp (-\alpha x)$, where $x$ is the length of the unpumped region between the end of the pump stripe and the edge of the sample and $\alpha$ is the loss coefficient.

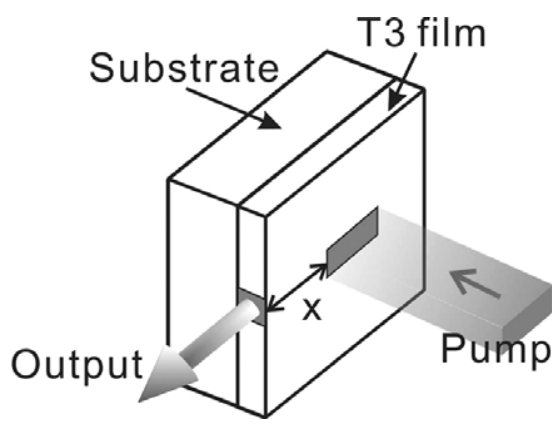

Fig. 4. Schematic setup for measurement of the waveguide loss. 


\section{Results and discussions}

\subsection{Thermal properties}

Fig. 5 shows the trace of the differential scanning calorimetry (DSC) of T3 molecules. The introduction of rigid aryl substitutions on $\mathrm{C} 9$ renders the molecular structure rather steric and bulky. As a consequence, T3 possesses a high glass transition temperature $\left(T_{\mathrm{g}}\right)$ of $\sim 200{ }^{\circ} \mathrm{C}$. Thus vacuum-deposited films of $\mathrm{T} 3$ are homogeneous and amorphous (as confirmed by X-ray diffraction), and stay morphologically stable well over $100{ }^{\circ} \mathrm{C}$. Interestingly, above $T_{\mathrm{g}}$, T3 does not show any sign of phase transitions (e.g., crystallization and melting, etc.) up to $400{ }^{\circ} \mathrm{C}$, indicating it be a truly amorphous material and be difficult to crystallize. Indeed, heating a T3 film at a temperature close to or well over its $T_{\mathrm{g}}$ also does not induce apparent changes that can be distinguished by optical microscopy. Further characterization of optical constants of T3 films with spectroscopic ellipsometry, however, reveals that annealing at temperatures close to or above $T_{\mathrm{g}}$ causes significant changes in microscopic morphology (i.e., anisotropic vs. isotropic).

\subsection{Optical constants and molecular orientation}

In examining optical anisotropy in both the transparent region and the absorption region of the spectrum, due to the high degree of correlation between model parameters for optically anisotropic films, more ellipsometric data other than those from typical reflection ellipsometry at variable angles are required to reduce correlation and to obtain correct results [27-30]. Typical approaches include taking

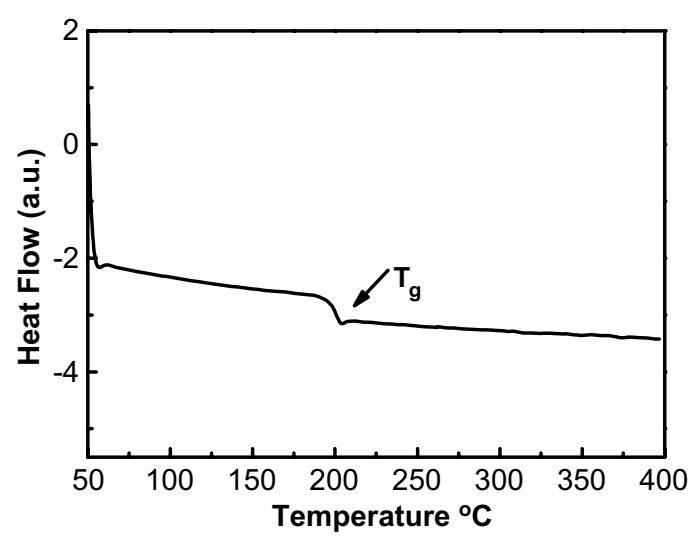

Fig. 5. The DSC trace of T3. data from samples of different thicknesses (i.e., multi-sample analysis), combining data from reflection ellipsometry and transmission measurements of a sample, or combining data from both reflection and transmission ellipsometry of a sample $[29,30]$. In this work, anisotropic optical constants of a sample were determined by the combination of reflection and transmission ellipsometry, which in principle is simpler and eliminates the risk of sample-to-sample variation (as may happen in the multi-sample analysis).

Ellipsometric data (both transmission and reflection) measured for a T3 film $(\sim 100 \mathrm{~nm}$ thick $)$ asdeposited and after annealing are shown in Figs. 6 and 7 , respectively. The annealing was performed at $200{ }^{\circ} \mathrm{C}$ for $4 \mathrm{~min}$ under a dry nitrogen atmosphere, and further annealing induced no more difference in optical properties. After annealing, the sample was then cooled by contact with a metal block held at the room temperature. As clearly
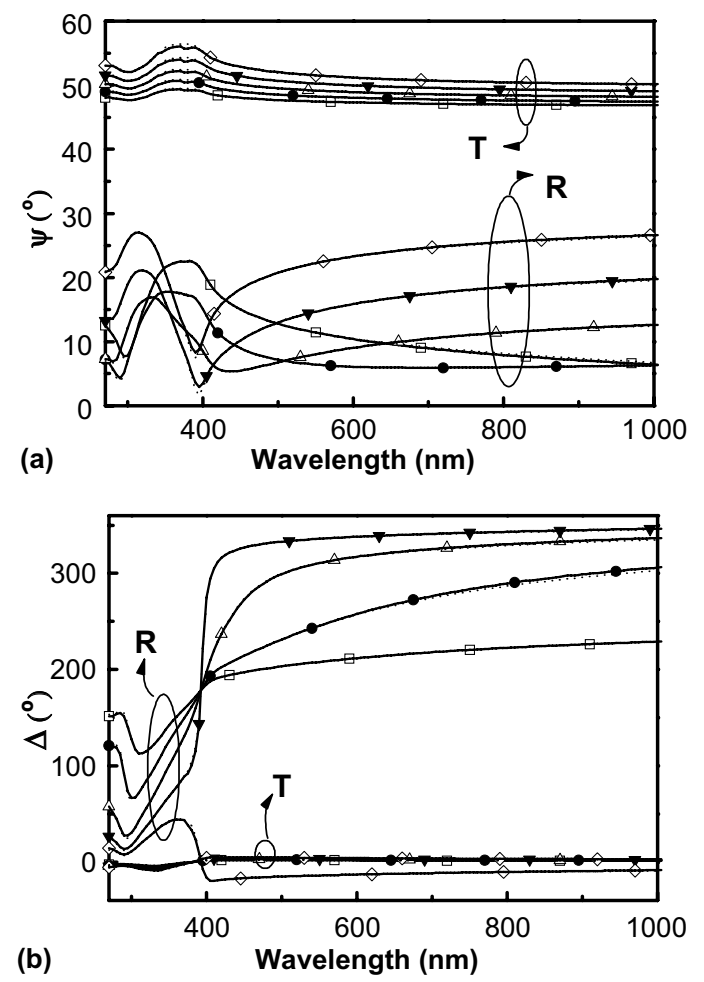

Fig. 6. Measured (solid lines) (a) $\Psi$ and (b) $\Delta$, and the fittings (dashed lines) for reflection ellipsometry $(\mathrm{R})$ at incident angles of $55^{\circ}(\square), 60^{\circ}(\bullet), 65^{\circ}(\triangle), 70^{\circ}(\nabla)$, and $75^{\circ}(\diamond)$ and for transmission ellipsometry $(\mathrm{T})$ at incident angles of $40^{\circ}(\square), 45^{\circ}(\bullet), 50^{\circ}$ $(\triangle), 55^{\circ}(\boldsymbol{\nabla})$, and $60^{\circ}(\diamond)$ of an as-deposited T3 film (100 nm). The fitted data (dashed lines) are difficult to identify because of the high quality of the fit. 

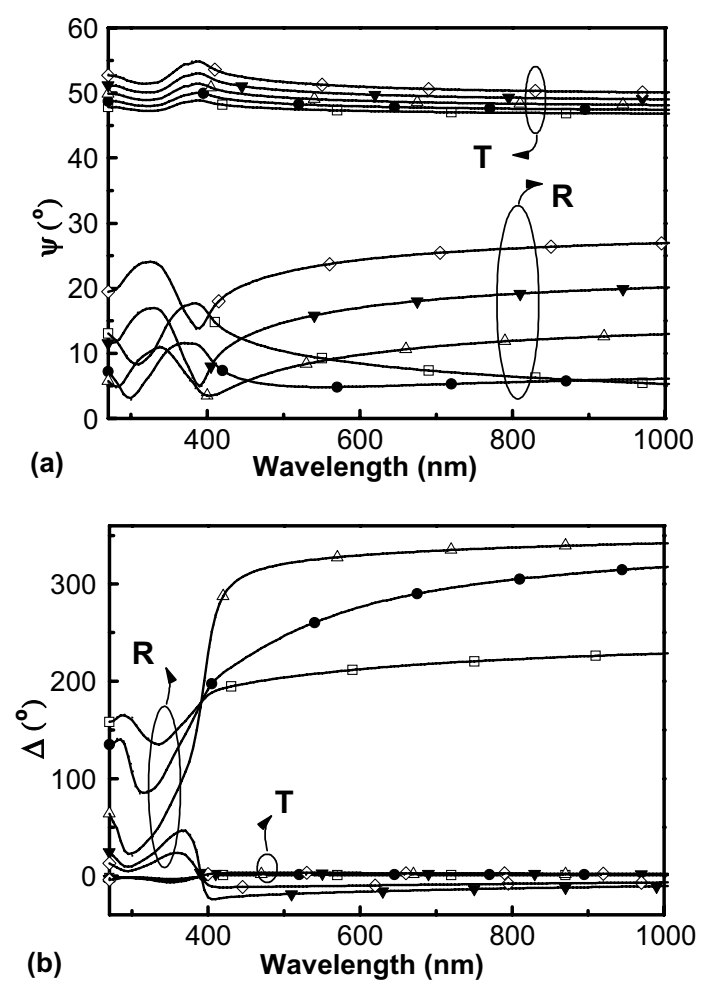

Fig. 7. Measured (solid lines) (a) $\Psi$ and (b) $\Delta$, and the fittings (dashed lines) for reflection ellipsometry (R) at incident angles of $55^{\circ}(\square), 60^{\circ}(\bullet), \quad 65^{\circ}(\triangle), 70^{\circ}(\mathbf{\nabla})$, and $75^{\circ}(\diamond)$, and for transmission ellipsometry (T) at incident angles of $40^{\circ}(\square)$, $45^{\circ}(\diamond), 50^{\circ}(\triangle), 55^{\circ}(\mathbf{\nabla})$, and $60^{\circ}(\diamond)$ of an annealed T3 film $(100 \mathrm{~nm})$. The fitted data (dashed lines) are difficult to identify because of the high quality of the fit.

shown in Figs. 6 and 7, ellipsometric values ( $\Psi$ and $\Delta$ ) change upon sample annealing. The alteration of such optical properties is not due to degradation or transformation of materials with annealing, since the PL spectra before and after annealing are basically the same and the variation of PL quantum yields as determined with the calibrated integrating sphere system is within the experimental error $(75 \pm 3 \%)$. Instead, such results indicate changes in microscopic morphology of the T3 film induced with annealing and the sensitivity of ellipsometry in detecting such characteristics.

Since no in-plane anisotropy was observed in polarized optical transmission microscopy and the ellipsometric data were the same when rotating the sample in the plane of the film, in extracting optical constants from the measured ellipsometric data, the films were treated as uniaxially anisotropic with the optical axis along the surface normal. That is, the optical constants are distinguished by those for the ordinary (in-plane) polarization, $n_{\mathrm{o}}+\mathrm{i} k_{\mathrm{o}}$, and those for the extraordinary (out-of-plane) polarization, $n_{\mathrm{e}}+\mathrm{i} k_{\mathrm{e}}$. The film thickness was first determined using a uniaxially anisotropic Cauchy model to fit the ellipsometric data in the transparent region. A Kramers-Kronig consistent model using a combination of one Cauchy background and several Gaussian oscillators for the ordinary (in-plane) and extraordinary (out-of-plane) optical constants was then constructed to fit the measured ellipsometric data over the whole spectral range [27-30]. Optical constants thus extracted (Figs. 8(a) and (b)) well fitted the experimental ellipsometric data with low MSE values of $\leqslant 2$, as illustrated in Figs. 6 and 7 .

As shown in Fig. 8(a), the as-deposited T3 film exhibits significant anisotropy in both refractive index and absorption. The large difference between $k_{0}$ and $k_{\mathrm{e}}(\sim 4.4: 1$ at the peak wavelength) indicates preferential orientations of vacuum-deposited T3 molecules parallel to the surface plane, since the transition dipole moment for the $\pi-\pi^{*}$ transition
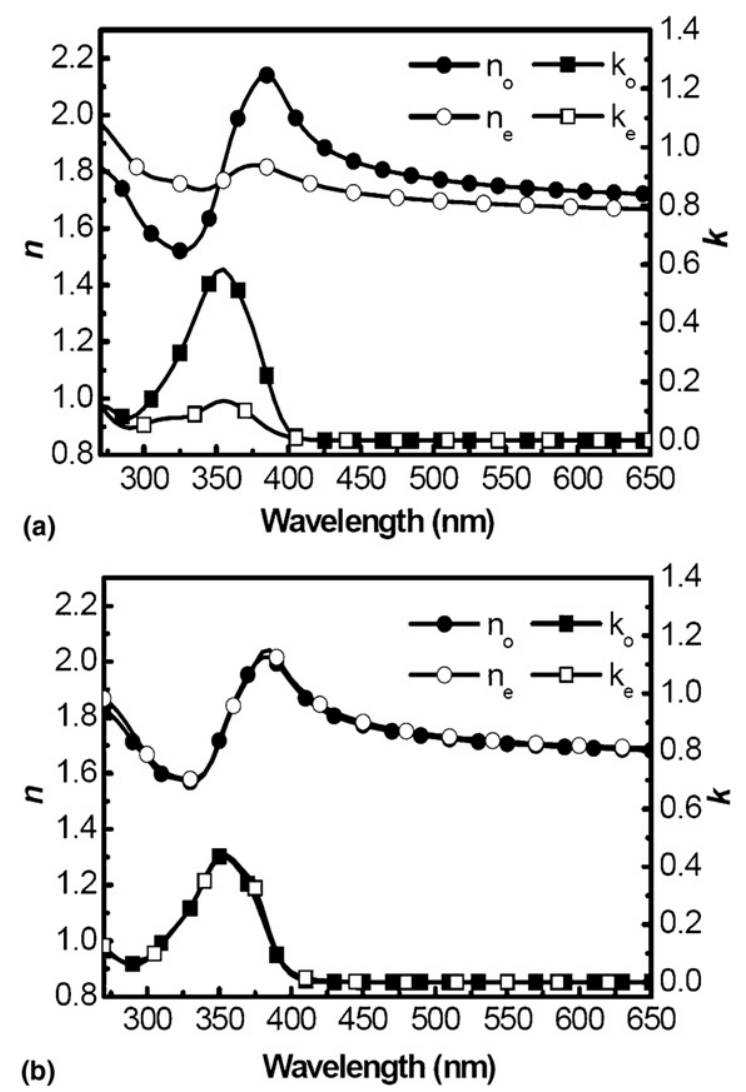

Fig. 8. Ordinary (in-plane) and extraordinary (out-of-plane) optical constants of (a) the as-deposited T3 film, and (b) the annealed T3 film $\left(200{ }^{\circ} \mathrm{C}, 4 \mathrm{~min}\right.$.). 
of oligofluorenes is roughly along the backbone [34]. The optical constants of the annealed T3 film are shown in Fig. 8(b). With annealing, the difference between in-plane and out-of-plane optical constants diminishes and the film becomes optically isotropic. This result suggests that $\mathrm{T} 3$ molecules reorient themselves during annealing and eventually take an isotropic orientational distribution, which is then frozen down to the room temperature with the cooling performed. Schematic representation of distributions of molecular orientation in the asdeposited and the annealed films are shown in Figs. 9(a) and (b), respectively. Such a phenomenon shall not be too surprising since $\mathrm{T} 3$ is an organic glass. For organic glasses, although there occurs no major morphological change and phase transition across $T_{\mathrm{g}}$, the viscosity in general would substantially decrease and molecules acquire larger freedom in motion and rotation [35]. Characteristics unique to T3 here, however, are its anisotropy as deposited and its immunity to crystallization above $T_{\mathrm{g}}$.

\subsection{Stimulated emission}

To study the influences of molecular orientational distributions on stimulated emission characteristics, ASE of the T3 film $(100 \mathrm{~nm})$ as deposited or after annealing was characterized. Figs. 10(a) and (b) show the edge-collected emission spectra from a T3 film as-deposited and after annealing, respectively, at different excitation intensities. At low pump intensities, the emission spectra for both
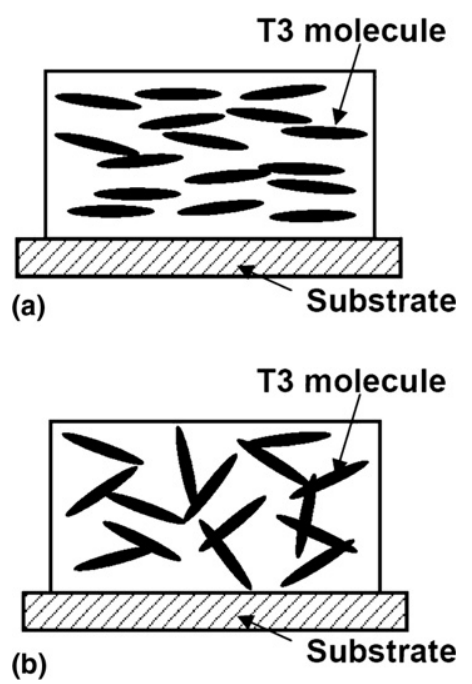

Fig. 9. Schematic illustration of distributions of molecular orientations in (a) the as-deposited and (b) the annealed T3 films.
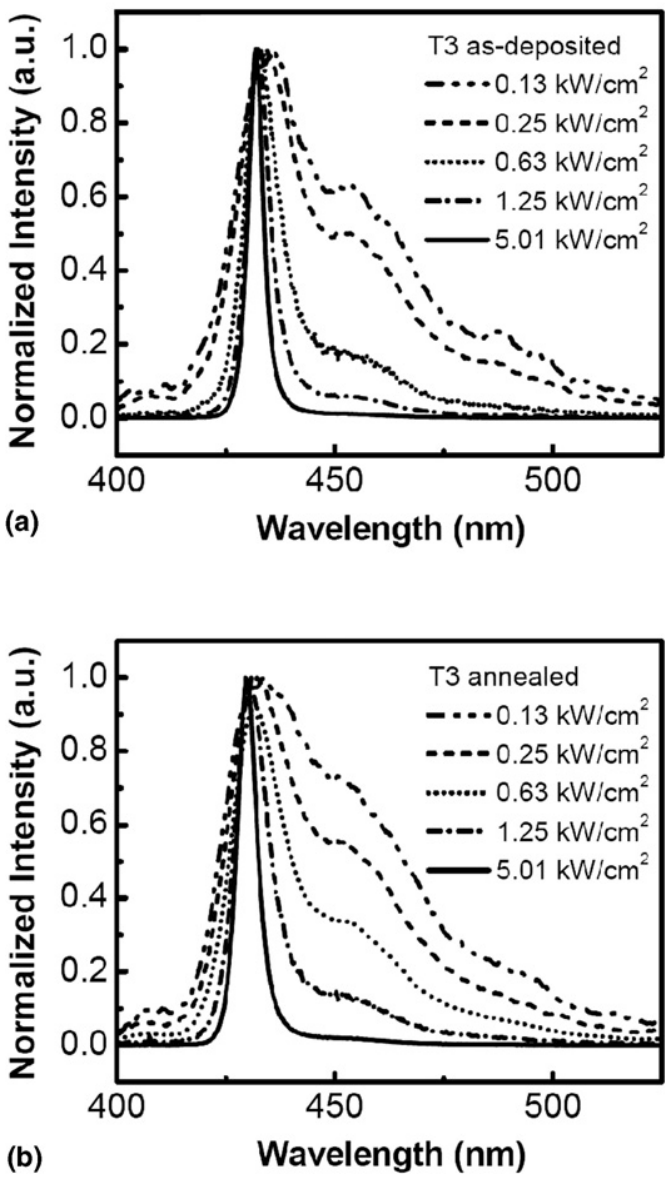

Fig. 10. Normalized edge-detected emission spectra of (a) the asdeposited T3 film, and (b) the annealed T3 film $\left(200^{\circ} \mathrm{C}, 4 \mathrm{~min}\right)$ at different pump intensities.

cases are broad and exhibit clear vibronic peaks and shoulders at $405 \mathrm{~nm}(0-0$ transition), $430 \mathrm{~nm}$ (0-1 transition), $455 \mathrm{~nm}$ (0-2 transition), and near $480 \mathrm{~nm}$ (0-3 transition), characteristic of spontaneous emission. As the pump energy is increased, the component associated with stimulated emission increases and results in gain narrowing centered at $\sim 430 \mathrm{~nm}$, which coincides with the $0-1$ vibronic transition of the T3 spontaneous emission. With increasing the pumping intensities, emission spectra of the as-deposited film narrow faster than those of the annealed film, indicating a lower threshold intensity required for observing ASE in the asdeposited film. Fig. 11(a) shows the corresponding full width at half maximum (FWHM) of emission spectra for these two cases as a function of the pump intensity. As the pump intensity is raised well beyond the threshold values, the intensity at the 430-nm peak grows much more than those at other 


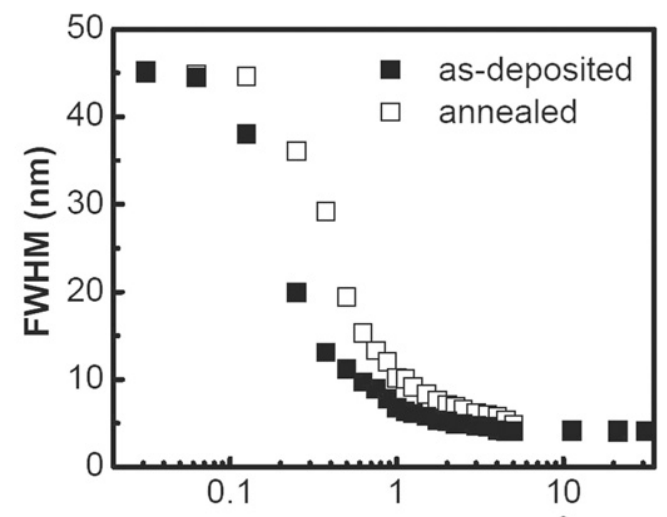

(a)

\section{Pump Intensity $\left(\mathrm{kW} / \mathrm{cm}^{2}\right)$}

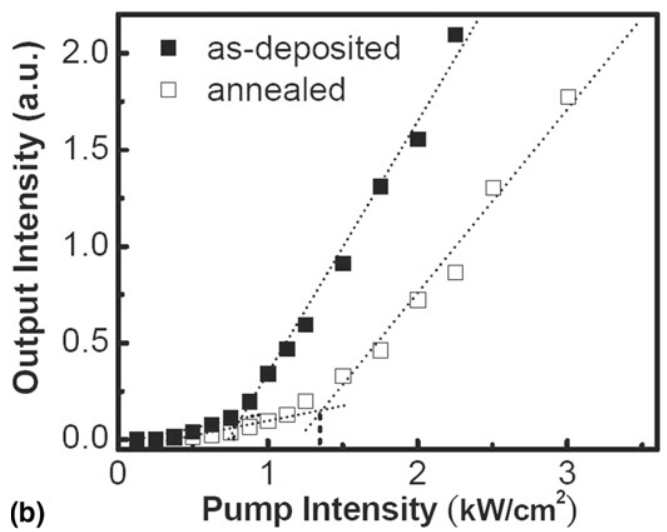

Fig. 11. (a) FWHM of edge-detected emission spectra, and (b) Edge-detected output intensity as a function of the pump intensity for the as-deposited T3 film and the annealed T3 film $\left(200{ }^{\circ} \mathrm{C}, 4 \mathrm{~min}\right.$.).

wavelengths, resulting in a largely reduced FWHM of $<5 \mathrm{~nm}$.

The spectral narrowing is accompanied by the abrupt increase of the edge-detected light intensity (at the ASE wavelength) vs. the excitation intensity, as shown in Fig. 11(b). The abrupt increase of edge emission indicates the onset of stimulated emission along the pumped waveguide region, and thus defines the threshold pump intensity for the ASE to occur. The threshold pump intensities for a film as-deposited and after annealing are $\sim 0.7 \mathrm{~kW} / \mathrm{cm}^{2}$ and $\sim 1.4 \mathrm{~kW} / \mathrm{cm}^{2}$, respectively, which correspond well to pump intensities where strong spectral narrowing occurs. It is noticed that the as-deposited T3 film exhibits a lower ASE threshold than the annealed film.

Stimulated emission of the as-deposited film and the annealed film were further characterized with the variable-stripe-length (VSL) technique (Fig. 3) to measure the net gain of ASE along the wave- guide. Fig. 12(a) and (b) show the output intensity at the ASE peak wavelength (symbols) for the asdeposited film and the annealed film, respectively, as a function of the pump stripe-length at different pump intensities. By fitting experimental data using Eq. (1) (represented by dashed lines), the net gain coefficients are determined and are shown in Fig. 13 for both cases. Note that at higher pump intensities, the output intensity saturates at longer excitation lengths. It is typically observed in other solid-state organic gain media as the strongly amplified traveling light induces substantial depletion of the excitations, resulting in gain saturation [36]. Therefore, in the fitting, only subsets of data $(g L<4)$ for which gain saturation is not evident are used. For the as-deposited T3 film, as pumping intensity increases from 1 to $30 \mathrm{~kW} / \mathrm{cm}^{2}$, the net gain increases by nearly two orders of magnitude from 2.6 to $78 \mathrm{~cm}^{-1}$. A net gain of $78 \mathrm{~cm}^{-1}$ achieved at a pump intensity of $\sim 30 \mathrm{~kW} / \mathrm{cm}^{2}$ is rather high among those reported for neat semiconducting
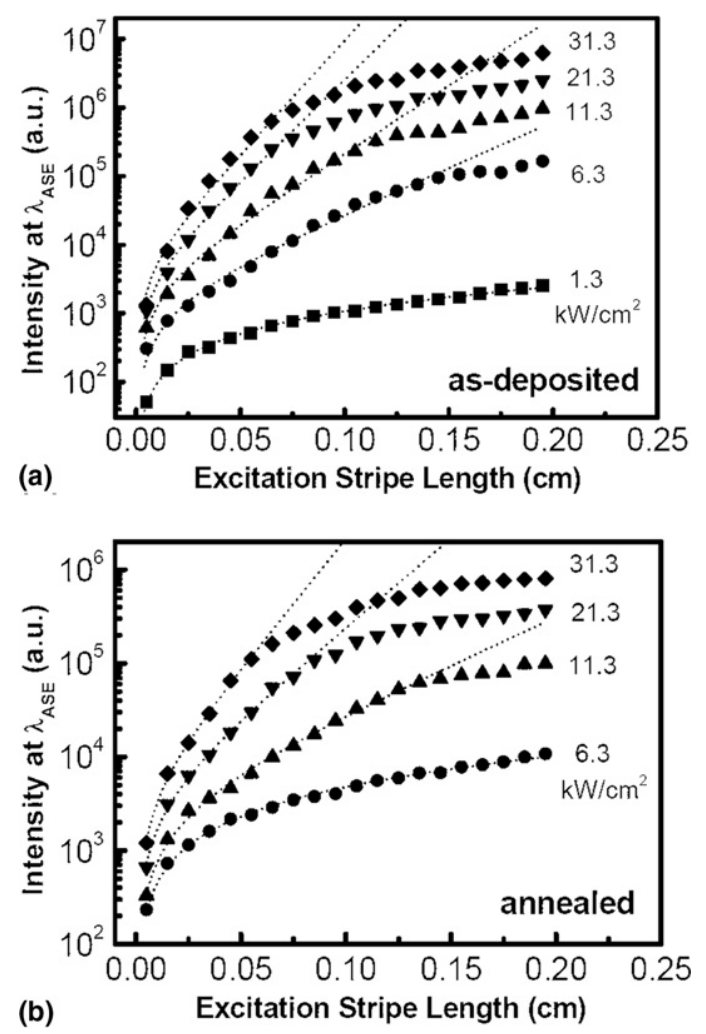

Fig. 12. Measured (symbols) and fitted (lines) emission intensities at the ASE wavelength (symbols) for (a) the as-deposited T3 film, and (b) the annealed $\mathrm{T} 3 \mathrm{film}\left(20{ }^{\circ} \mathrm{C}, 4 \mathrm{~min}\right.$.) as a function the pump length at several pump intensities. 


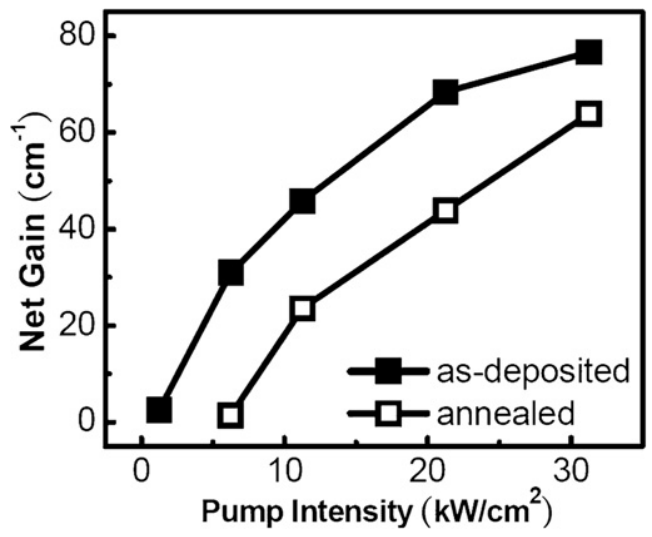

Fig. 13. Net gain coefficients as a function of the pump intensity for the as-deposited and the annealed T3 films.

organic films [31-33]. After annealing, at a same pumping intensity, the net gain becomes substantially lower $\left(15-30 \mathrm{~cm}^{-1}\right.$ lower). Such lower net gains explain the higher ASE threshold for the annealed T3 films.

The loss characteristics of the waveguides were also measured using the scheme described in Section 2. Fig. 14 shows the edge-detected emission intensity at the ASE wavelength as a function of the stripe distance from the waveguide edge. By curve fitting the experimental data with an exponential dependence on length, loss coefficients of $7.9 \mathrm{~cm}^{-1}$ and $5.7 \mathrm{~cm}^{-1}$ are extracted for the as-deposited and the annealed films, respectively. Such values are rather small for neat organic films [31-33], and are close to those reported for host-guest systems in which

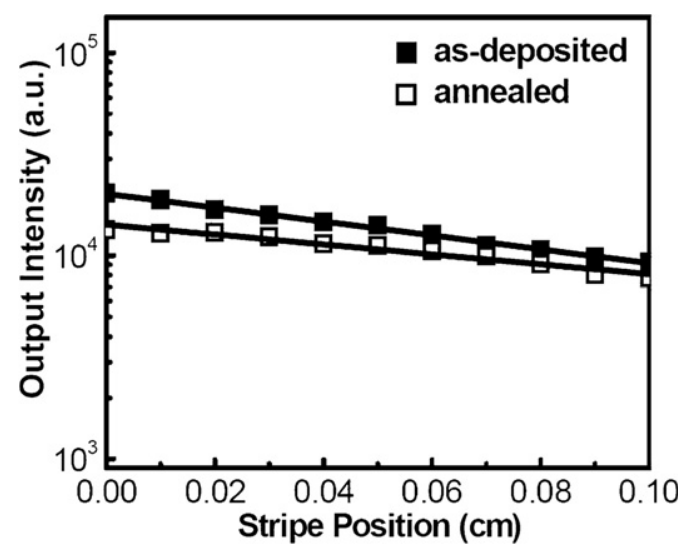

Fig. 14. Measured (symbols) and fitted (lines) edge-detected emission intensities for the as-deposited T3 film and the annealed T3 film as a function of the distance between the end of the excitation stripe and the edge of the substrate. energy transfer moves the emission away from the absorption tail $[9,10]$. The slightly lower loss coefficient for the annealed film is due to the lower in-plane absorption (compared to the anisotropic as-deposited film), which would reduce loss resulted from self-absorption.

The lower ASE threshold and the higher net gain of stimulated emission in the anisotropic T3 film than in the isotropic T3 film are worthy of some considerations. To the first order (i.e., neglecting the waveguide loss), the net gain coefficient $\mathrm{g}$ roughly has the dependence of $g=\Gamma \times g^{\prime}$, where $g^{\prime}$ is the intrinsic material gain and $\Gamma$ is confinement ratio for the waveguide mode supported by the slab waveguide (i.e., the fundamental transverse electric (TE) mode in the present case) $[18,36,37]$. As the uniaxially anisotropic T3 film has a higher in-plane refractive index than the isotropic film (by $\sim 0.1-$ 0.15 at emission wavelengths), the waveguide mode (i.e., the fundamental TE mode which has an inplane polarization) in the anisotropic $\mathrm{T} 3$ film sees a larger confinement. As to $g^{\prime}$, it has the general dependence of $g^{\prime}=\sigma_{\mathrm{em}} \times N_{\mathrm{ex}}$, where $\sigma_{\mathrm{em}}$ is the cross section for stimulated emission and $N_{\text {ex }}$ represents the population of molecular excited states [38-40]. With nearly same film thicknesses and the stronger in-plane extinction coefficient, the anisotropic T3 film has a slightly higher absorption of the incident excitation than the isotropic film $(77 \%$ vs. $72 \%)$ and thus slightly higher $N_{\mathrm{ex}}$. Since $\sigma_{\mathrm{em}}$ depends on the alignment of the molecular transition dipole moments with the polarization of stimulated emission (i.e., the polarization of the fundamental TE mode) [38], the anisotropic T3 film which has the transition dipole moments preferentially along the surface also has larger $\sigma_{\mathrm{em}}$. These factors all thus favor a larger net gain of stimulated emission in the anisotropic T3 film even though it has a slightly higher waveguide loss. The results therefore suggest that if in the thin film molecules and their transition dipole moments can be well aligned with the polarization of stimulated emission yet without causing significant loss and emission quenching, the gain and threshold of stimulated emission could be substantially enhanced beyond the isotropic case.

\section{Summary}

In summary, the efficient luminescence and interesting thermal properties of oligofluorenes provide the opportunity to investigate influences of molecular orientational distributions on characteristics of 
stimulated emission in organic semiconducting films. Vacuum-deposited terfluorene films exhibit preferential in-plane molecular orientation, yet with annealing around or above its $T_{\mathrm{g}}$, the molecules reorient themselves to become isotropic, as examined by spectroscopic ellipsometry. Detailed studies of stimulated emission characteristics, including spectra, intensity, gain and loss, of both the anisotropic and the isotropic films have been performed. Results show that the anisotropic terfluorene films exhibit higher gain for stimulated emission and lower ASE threshold than the isotropic films, due to larger confinement of the waveguided mode and larger cross section of stimulated emission. A net gain as high as $78 \mathrm{~cm}^{-1}$ (at a pump intensity of $\sim 30 \mathrm{~kW} / \mathrm{cm}^{2}$ ), an ASE threshold as low as $0.7 \mathrm{~kW} / \mathrm{cm}^{2}$, and low loss coefficients of $5-8 \mathrm{~cm}^{-1}$ for the waveguide were observed. The results suggest that the gain and threshold of stimulated emission in organic semiconducting films could be substantially improved beyond the isotropic case if molecules and their transition dipole moments can be well aligned with the polarization of stimulated emission yet without causing significant loss and emission quenching.

\section{Acknowledgements}

The authors would like to acknowledge financial support from National Science Council of Republic of China. C.-L.L. and H.-W.L are also grateful for financial support from MediaTek Fellowship.

\section{References}

[1] C.W. Tang, S.A. VanSlyke, Appl. Phys. Lett. 51 (1987) 913.

[2] C.W. Tang, Appl. Phys. Lett. 48 (1986) 183.

[3] J.H. Burroughes, D.D.C. Bradley, A.R. Brown, R.N. Marks, R.H. Friend, P.L. Burns, A.B. Holmes, Nature (London) 347 (1990) 539.

[4] A.A. Shoustikov, Y. You, M.E. Thompson, IEEE J. Sel. Top. Quantum Electron. 4 (1998) 3.

[5] F. Garnier, G. Horowitz, X. Peng, D. Fichou, Adv. Mater. 2 (1990) 592.

[6] T.N. Jackson, Y.-Y. Lin, D.J. Gundlach, H. Klauk, IEEE J. Sel. Top. Quantum Electron. 4 (1998) 100.

[7] F. Hide, M.A. Diáz-Garcia, B.J. Schwartz, M.R. Andersson, Q. Pei, A.J. Heeger, Science 273 (1996) 1833.

[8] N. Tessler, G.J. Denton, R.H. Friend, Nature (London) 382 (1996) 695.

[9] V.G. Kozlov, V. Bulovic, P.E. Burrows, S.R. Forrest, Nature (London) 389 (1997) 362.

[10] M. Berggren, A. Dodabalapur, R.E. Slusher, Z. Bao, Nature (London) 389 (1997) 466.
[11] G.J. Denton, N. Tessler, N.T. Harrison, R.H. Friend, Phys. Rev. Lett. 78 (1997) 733.

[12] S.V. Frolov, M. Liess, P.A. Lane, W. Gellerman, Z.V. Vardeny, M. Ozaki, K. Yoshino, Phys. Rev. Lett. 78 (1997) 4285 .

[13] N.D. Kumar, J.D. Bhawalkar, P.N. Prasad, F.E. Karasz, B. Hu, Appl. Phys. Lett. 71 (1997) 999.

[14] V.G. Kozlov, P.E. Burrows, G. Parthasarathy, Appl. Phys. Lett. 74 (1999) 1057.

[15] M.A. Baldo, R.J. Holmes, S.R. Forrest, Phys. Rev. B 66 (2002) 035321.

[16] H. Yamamoto, T. Oyamada, H. Sasabe, C. Adachi, Appl. Phys. Lett. 84 (2004) 1401.

[17] T. Aimono, Y. Kawamura, K. Goushi, H. Yamamoto, H. Sasabe, C. Adachi, Appl. Phys. Lett. 86 (2005) 071110.

[18] M. Pauchard, M. Vehse, J. Swensen, D. Moses, A.J. Heeger, E. Perzon, M.R. Andersson, Appl. Phys. Lett. 83 (2003) 4488.

[19] K.-T. Wong, Y.-Y. Chien, R.-T. Chen, C.-F. Wang, Y.-T. Lin, H.-H. Chiang, P.-Y. Hsieh, C.-C. Wu, C.-H. Chou, Y.-O. Su, G.-H. Lee, S.-M. Peng, J. Am. Chem. Soc. 124 (2002) 11576.

[20] C.C. Wu, T.L. Liu, W.Y. Hung, Y.T. Lin, K.T. Wong, R.T. Chen, Y.M. Chen, Y.Y. Chien, J. Am. Chem. Soc. 125 (2003) 3710.

[21] C.-C. Wu, T.-L. Liu, Y.-T. Lin, W.-Y. Hung, T.-H. Ke, K.-T. Wong, T.-C. Chao, Appl. Phys. Lett. 85 (2004) 1172.

[22] C.-C. Wu, Y.-T. Lin, K.-T. Wong, R.-T. Chen, Y.-Y. Chien, Adv. Mater. 16 (2004) 61.

[23] H.W. Lin, C.L. Lin, C.C. Wu, T.C. Chao, K.T. Wong, Appl. Phys. Lett. 87 (2005) 071910.

[24] L.-Y. Chen, W.-Y. Hung, Y.-T. Lin, C.-C. Wu, Appl. Phys. Lett. 87 (2005) 112103.

[25] C.-C. Wu, W.-G. Liu, W.-Y. Hung, T.-L. Liu, Y.-T. Lin, H.-W. Lin, K.-T. Wong, Y.-Y. Chien, R.-T. Chen, T.-H. Hung, Appl. Phys. Lett. 87 (2005) 052103.

[26] C.-C. Wu, W.-Y. Hung, T.-L. Liu, L.-Z. Zhang, T.-Y. Luh, J. Appl. Phys. 93 (2003) 5465.

[27] R.M.A. Azzam, N.M. Bashara, Ellipsometry and Polarized Light, Amsterdam, North-Holland, 1977.

[28] G.E. Jellison, Thin Solid Films 313-314 (1998) 33.

[29] M. Tammer, A.P. Monkman, Adv. Mater. 14 (2002) 210.

[30] C.M. Ramsdale, N.C. Greenham, Adv. Mater. 14 (2002) 212.

[31] M.D. McGehee, R. Gupta, S. Veenstra, E.K. Miller, M.A. Diaz-Garcia, A.J. Heeger, Phys. Rev. B58 (1998) 7035.

[32] G. Heliotis, D.D.C. Bradley, G.A. Turnbull, I.D.W. Samuel, Appl. Phys. Lett. 81 (2002) 415.

[33] R. Xia, G. Heliotis, D.D.C. Bradley, Appl. Phys. Lett. 82 (2003) 3599.

[34] Y. Geng, A. Trajkovska, D. Katsis, J.J. Ou, S.W. Culligan, S.H. Chen, J. Am. Chem. Soc. 124 (2002) 8337.

[35] K. Naito, A. Miura, J. Phys. Chem. 97 (1993) 6240.

[36] A. Yariv, Quantum Electronics, Wiley, New York, 1975.

[37] M. Pauchard, J. Swensen, D. Moses, A.J. Heeger, E. Perzon, M.R. Andersson, J. Appl. Phys. 94 (2003) 3543.

[38] K. Shimoda, Introduction to Laser Physics, Spinger-Verlag, Berlin Heidelberg, 1986.

[39] X. Liu, C. Py, Y. Tao, Y. Li, J. Ding, M. Day, Appl. Phys. Lett. 84 (2004) 2727.

[40] W. Holzer, A. Penzkofer, S.-H. Gong, A. Bleyer, D.D.C. Bradley, Adv. Mater. 8 (1996) 974. 